\title{
Decision aids reduced decisional conflict in patients with newly diagnosed hypertension
}

Montgomery AA, Fahey T, Peters TJ. A factorial randomised controlled trial of decision analysis and an information video plus leaflet for newly diagnosed hypertensive patients. Br J Gen Pract 2003;53:446-53.

\section{Do simple or complex decision aids assist patients with newly diagnosed hypertension in deciding whether to start drug treatment?}

METHODS

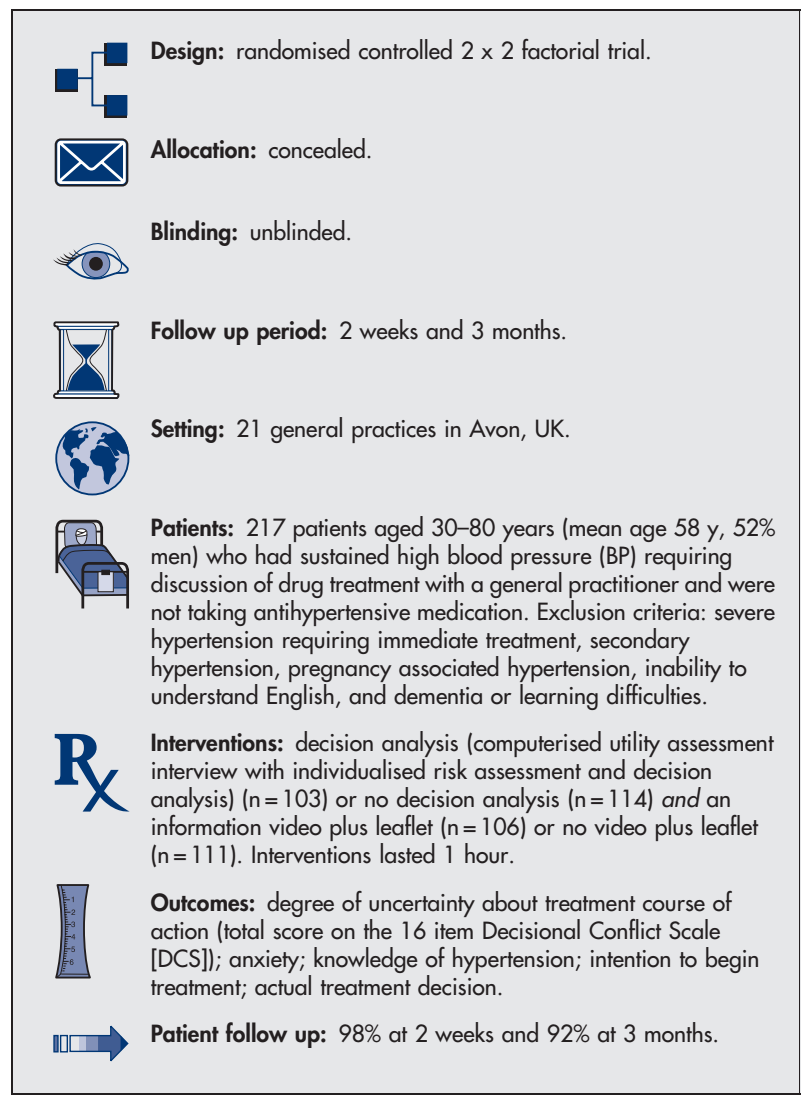

\section{MAIN RESULTS}

Analysis was by intention to treat. At 3 months, 133 patients (67\%) had been prescribed antihypertensives.

Decision analysis $v$ no decision analysis and video plus leaflet $v$ no video plus leaflet for decisional conflict in newly diagnosed hypertension at 2 weeks*

\begin{tabular}{|c|c|c|}
\hline Comparison & Mean total score & Adjusted difference $(95 \% \mathrm{Cl}) \dagger$ \\
\hline $\begin{array}{l}\text { Decision analysis } v \\
\text { no decision analysis } \\
\text { Video plus leaflet } v \\
\text { no video plus leaflet }\end{array}$ & $\begin{array}{l}27.6 \vee 38.9 \\
30.3 \vee 36.8\end{array}$ & $\begin{array}{l}-9.4(-13.0 \text { to }-5.8) \\
-4.2(-7.8 \text { to }-0.6)\end{array}$ \\
\hline \multicolumn{3}{|c|}{$\begin{array}{l}{ }^{*} \mathrm{Cl} \text { defined in glossary. } \\
\dagger \text { Adjusted for age, sex, decisional conflict at baseline, factorial design, } \\
\text { and general practice. }\end{array}$} \\
\hline
\end{tabular}

........................... For correspondence: Dr A A Montgomery, Division of Primary Health Care, University of Bristol, Bristol, UK. alan.a.montgomery@bristol.ac.uk Source of funding: UK Medical Research Council.
At 2 weeks, patients who received decision analysis had less decisional conflict than patients who did not receive decision analysis (table); the groups did not differ for anxiety levels (mean score $34.8 \mathrm{v}$ 36.8 out of 80 , adjusted difference $-2.8,95 \% \mathrm{CI}-5.6$ to 0.1 ), intention to begin treatment (yes $v$ unsure adjusted risk ratio 1.19 , CI 0.59 to 2.40 ); no $v$ unsure adjusted risk ratio 3.15 , CI 0.91 to 10.98 ), or actual treatment decision (medication prescribed $67.7 \% \vee 66.0 \%$, adjusted odds ratio 1.13 , CI 0.59 to 2.19). Similar results were found for patients who received the video plus leaflet compared with those who did not.

Patients who received decision analysis as well as the video plus leaflet had less decisional conflict (unadjusted mean score 27.1) than patients who received decision analysis alone (28.2), video plus leaflet alone (33.3), or no intervention (44.2). Decision analysis and the video plus leaflet interacted (interaction coefficient $12.5,95 \%$ CI 5.4 to $19.5, p=0.001$ for decisional conflict), suggesting a ceiling to the amount of information from which patients can benefit.

\section{CONCLUSION}

Simple (video plus leaflet) or complex (decision analysis) decision aids each reduced decisional conflict in patients with newly diagnosed hypertension, but did not affect anxiety, intention to start antihypertensive treatment, or the actual treatment decision.

\section{A modified version of this abstract appears in Evidence-Based Medicine}

\section{Commentary}

ncreasingly, patients are expected to have a greater role in healthcare decision making. Through increased knowledge, realistic expectations, and structured treatment options, decision aids can help increase patient participation.

Montgomery et al evaluated 2 interventions to aid patients' hypertension treatment decisions, and found that patients who used decision aids better understood their condition and had lower levels of decisional conflict. This finding implies that they were more confident in their decision choices.

As a safeguard against poor quality advice, decision aids should be based on robust research evidence. The quality of the evidence underpinning the interventions in the study by Montgomery et al is difficult to judge. The use of a factorial design in this study confers the advantage of efficiency by allowing 2 trials within a single sample as a 2 arm trial. In this study, however, there was an interaction between the video/leaflet and the decision analysis, such that the effect of each intervention was reduced in the presence of the other. Despite reducing conflict, improving knowledge, and increasing confidence, the use of decision aids did not alter the actual decisions made. This suggests that the benefits of decision aids are limited primarily to conflict resolution or that the decision choices already considered by the patient concurred with the results of the decision analysis. The clinical significance of any gains is unclear. Without an economic analysis of the costs associated with the benefits, it is difficult to judge the overall benefits of the decision aids assessed in this study.

Ultimately, if decision support helps patients become more involved in the decision making process, long term benefits such as informed patients who understand their condition and are confident in their treatment decisions may outweigh the costs.

Anne Taylor, RGN Department of Nursing and Midwifery, University of Stirling Stirling, UK

Carl Thompson, PhD, RN Department of Health Sciences, University of York York, UK

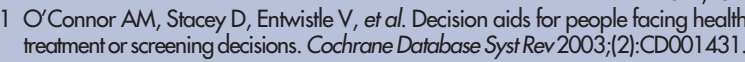

